\title{
Harm reduction policy
}

Addiction Ontology

\section{Source}

Addiction Ontology

Definition: A social policy whose aim is to reduce the harm to a population from continued use of a psychoactive substance by members of that population.

Comment: This entity is defined in terms of the aims of a policy rather than its effect. It is distinguished from harm reduction behaviour. It includes attempting to get people to reduce their use of the substance, change their pattern of use, change the type or formulation of the substance they consume. Note that the substance does not just include the psychoactive drug; it can include whole commodities such as tobacco.

This definition was imported from the Addiction Ontology (see https://addictovocab.org/). Comments and suggestions for improvements are welcome using the Qeios review system.

Definitions imported from the Addiction Ontology (AddictO) are what are known as 'ontological definitions'. See this article in Qeios for an explanation https://www.qeios.com/read/YGIF9B.

Ontological definitions can sometimes be hard to read. In those cases we also include an informal definition.

Definitions also often require elaboration to make it clear how they should be used and what they include. In those cases we include a comment.

Also, definitions sometimes require an explanation as to how they came about to help users understand how they relate to alternative definitions. In those cases we include a curator note. 